\title{
Transition accomplished New setting and new structure of PAGES
}

\author{
Thorsten Kiefer', H. Fischer', A. Mix ${ }^{3}$, S. Fritz ${ }^{4}$, L. von Gunten ${ }^{5}$ and L. Goodwin ${ }^{5}$
}

\begin{abstract}
PAGES has revised its scientific structure to better align with Future Earth's philosophies of increasing interdisciplinarity and societally-relevant science. The new structure represents an integrative space between climate, environment, and humans as the key components of a sustainable Earth system.
\end{abstract}

The landscape of global change programs within which PAGES is embedded is transforming, as previous articles highlighted already (PAGES IPO 2014). The International Geosphere-Biosphere Programme (IGBP), PAGES' umbrella organization since its inception in 1991, will conclude by the end of 2015. IGBP's legacy and that of two other global change programmes, namely Diversitas and the International Human Dimensions Programme, will transfer to Future Earth, a large research platform for global sustainability that has been developing over the last few years.

Future Earth, subtitled "research for global sustainability", aims to provide the knowledge and support to accelerate our transformations to a sustainable world. To reach this ambitious goal, Future Earth intends to modify the approach taken by the predecessor programs in essentially three ways: (1) by re-directing research from addressing problems towards informing solutions to pressing societal issues, (2) by increasing the level of interdisciplinarity to an inclusiveness that reaches from the physical sciences, through the biogeochemical and ecological sciences to social sciences and the humanities, and (3) by working together with societal partners who have an interest in sustainability science (stakeholders), particularly in jointly designing initial research plans and end products.

From 2016, the former four global environmental change programs will have been boiled down into two. Future Earth will be complemented by the World Climate Research Programme (WCRP), which has formed a strong partnership with Future Earth, but will otherwise continue to coordinate its own international climate research.

In the handover from IGBP to Future Earth, IGBP's core projects, including PAGES, were invited to join the new initiative. PAGES' Scientific Steering Committee decided that PAGES would join Future Earth as a core project, while also broadening its thematic basis by establishing a scientific partnership with WCRP. Both affiliations have been formalized over recent months.

An open theme space replaces distinct foci Since the community consultations and plenary discussions at the PAGES Open Science Meeting in 2013 in Goa, PAGES has been preparing the terrain to successfully continue the facilitation of international scientific collaboration on past global changes, while

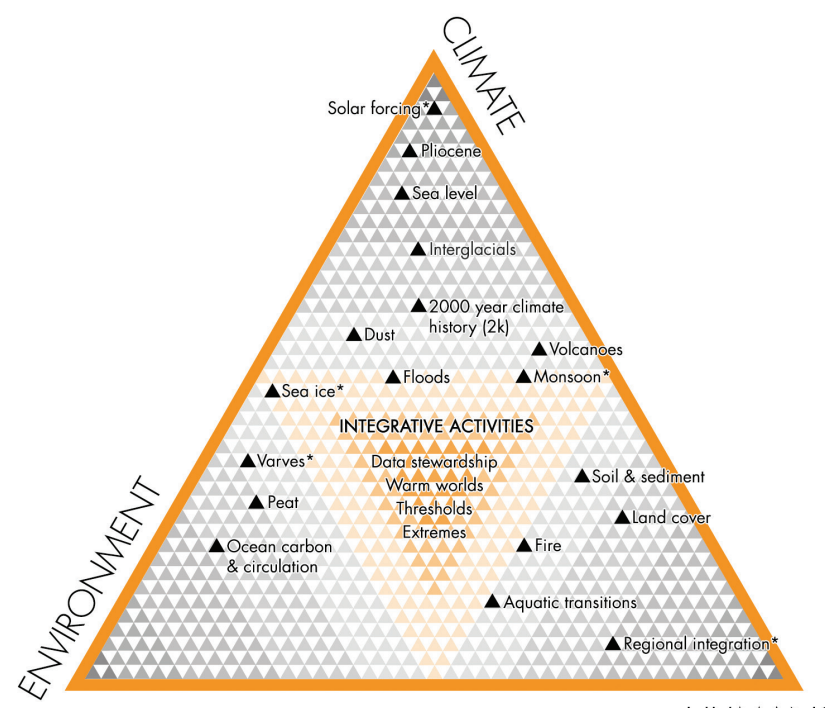

Figure 1: PAGES' science structure addresses the key components of the Earth system through the themes Climate, Environment, and Humans. The center of the triangle will hold cross-topical integrative activities, particularly suited to collaboration outside the PAGES community. Current working groups and the integrative activities are mapped on to the new science structure. The working groups marked with an asterisk are in their synthesis phase. also being able to link in with the agendas of Future Earth and WCRP.

Accordingly, PAGES has revised its scientific structure to better align with Future Earth's philosophies of integration and societally-relevant science. PAGES' science structure addresses the key components of the Earth system through the themes Climate, Environment, and Humans (Fig. 1). These themes define our scientific scope and reflect the holistic Earth system science approach that is becoming increasingly integrated within PAGES.

- The Climate theme represents quantitative climate system dynamics from a paleo-perspective. The aims are to improve knowledge on climate forcings, sensitivity, variability, modes, non-linearities and thresholds, Earth system feedbacks, regional-scale dynamics, and how current climate models represent the various aspects of climate dynamics.

- The Environment theme addresses components of the biosphere that interact with each other and climate, and may introduce feedbacks into the Earth system. This includes biogeochemical cycling, ecosystem dynamics, and ecosystem services.

- The Humans theme addresses long-term environmental changes where humans are a major agent, e.g. through land use, pollution, fertilization, soil erosion, river damming, or landscape fragmentation, and where environmental changes have a demonstrable effect on the functioning and well-being of ecosystem services and societies.

At first glance, the change from the previous circular structure to a triangular one might not look like a major coup. And indeed, what matters most in PAGES will remain, that our working groups are excellent, active, and productive. However, the modification of the structural geometry does actually go along with changes in emphasis and conceptual thinking within PAGES.

Replacing the four foci-climate forcings, regional climate, earth system dynamics, and human-climate-ecosystem interactions - with the cornerstones of the triangle has shifted the thematic emphasis away from its dominant climate focus towards giving more 
equal importance to human, environmental, and climatic impacts within the framework of fundamental Earth system science.

Another measure to streamline the structure was to deemphasize research on methods by eliminating the four former cross-cutting themes on chronology, proxies, modeling, and data management. This does not mean that methodological aspects are considered unimportant. However, research on chronology, proxies, and modeling in PAGES will need to be pursued in the context of addressing specific Earth system science questions. And the data management topic has even been identified as a matter of vital importance and has hence been further highlighted in the new structure (see Fig. 2).

The most fundamental change in philosophy reflected by the new structure might be that it eliminates the previous silo-like foci by streamlining them into themes that define a boundary-free, integrative scope. Accordingly, working groups do not need to be categorized any more as belonging to one particular focus or cross-cutting theme. Instead, the open thematic space now acknowledges and encourages that all working groups and other activities address a mixture of climatic, environmental, and human aspects. Graphically, the relative importance of those aspects places each working group in a certain region of the triangle (Fig. 2).

Finally, topics that are particularly integrative between climate, environment, and humans, are now centrally placed at the heart of PAGES rather than falling between the cracks of the former foci.

\section{A new layer of integration across working groups}

Many issues of concern to society cut across the specific topics addressed in working groups under the three new themes and will benefit from being addressed with an inclusive approach. The cross-topical integrative activities, depicted in the middle of the triangle, are thus a new format in PAGES dedicated to facilitating an additional level of scientific exchange, synthesis, and outreach. They build on the results and specialist expertise of the working groups and draw on complementary scientific expertise on modern process and future projection research from the networks of Future Earth and WCRP. Outputs are expected to provide information for impact assessments and management strategies and thus to form key contributions to the scientific agendas of Future Earth and WCRP.

At present, four integrative activities with the potential to provide transformative advances in approaches to paleoscience have been identified:

- Data stewardship: PAGES aims to develop sets of best practice guidelines for data standards, archiving, and access. This will be done by soliciting broad input from the international community of experts.

- Warm worlds: As the instrumental record fails to provide estimates related to future

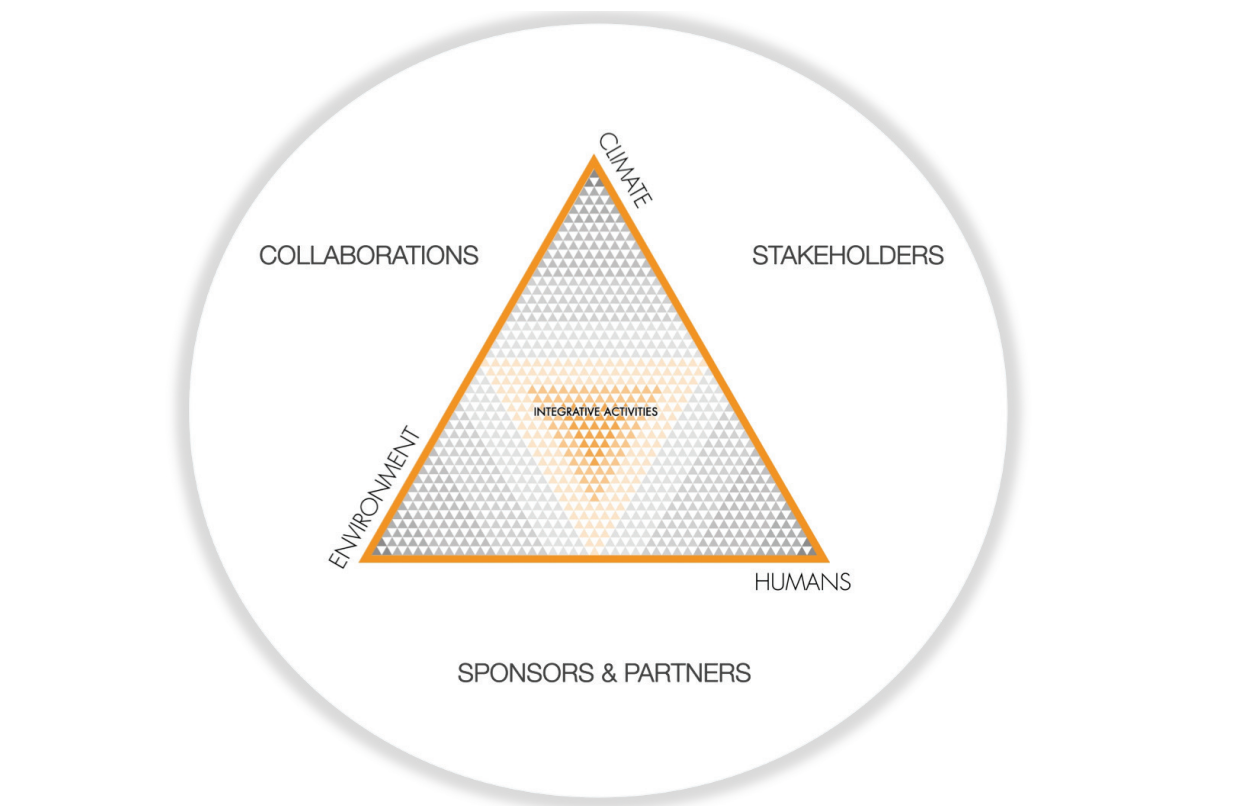

Figure 2: The PAGES science is embedded in a universe of opportunities for collaborations with the potential to enrich the diversity of research, impact of outreach, level of funding, and other aspects of the implementation of PAGES' agenda.

scenarios of a world that will be warmer than today by several degrees, PAGES aims to synthesize evidence for global and regional changes in the Earth system associated with warmer periods in the past. This also entails constraining the variability of natural systems under different conditions as baselines for assessing anthropogenic impact.

- Thresholds: This activity aims to synthesize the latest insights into the existence (or lack thereof) of multiple equilibria, including thresholds and tipping points in components of the Earth system.

- Extremes: A robust risk assessment of the probability of extreme events requires extending the data record of variability beyond the instrumental and documentary time period. This activity aims to identify the diverse range of paleoclimatic and paleoecological extreme events in the Earth system to derive comparable and statistically robust probability estimates of extreme events.

\section{Streamlining on the inside, opening to the outside}

The changes in scientific structure are accompanied by an evolution of internal and external organization. Internally, the working groups will remain the powerhouses of community-driven science in PAGES. To make them even more dynamic, they have gradually been streamlined in recent years by pacing their workplans into 3-year phases that conclude with syntheses.

A new management format will be required for the integrative activities. Combining the expertise from diverse working groups and loosely connected external communities will require that our Scientific Steering Committee members take on more active leadership roles and the PAGES office takes on more coordination than for the regular working groups.
The new program affiliations with Future Earth and WCRP are likely to entail more diverse and active connections to external groups of scientists and practitioners. The networks of the two programs extend the range of potential scientific contacts from the geosphere-biosphere community of IGBP to the entire range of sustainability science disciplines. Moreover, both Future Earth and WCRP will offer opportunities for highly interdisciplinary collaborative research, which should foster active collaborations across PAGES and its sister projects as well as other organizations.

Finally, a major component of Future Earth's approach to increasing the impact of science and supporting necessary transitions towards sustainability is its emphasis on collaboration with stakeholders. Core projects like PAGES are also called on to experiment with stakeholder engagement by developing approaches and building stakeholder networks. This might seem like a daunting task, but PAGES has already carried out an initial stakeholder analysis and added an encouragement to involve stakeholders to its workshop and working group proposal guidelines. We can thus expect that the orbit (Fig. 2) around the core of PAGES will rapidly populate with new people, new organizations, and new stimulating thinking.

\section{AFFILIATIONS}

PAGES International Project Office, Bern, Switzerland, now at Future Earth Global Hub, Paris, France ${ }^{2}$ Climate and Environmental Physics, University of Bern, Switzerland

${ }^{3}$ College of Earth, Ocean, and Atmospheric Sciences, Oregon State University, Corvallis, USA

${ }^{4}$ Department of Earth and Atmospheric Sciences,

University of Nebraska, Lincoln, USA

5PAGES International Project Office, Bern, Switzerland

CONTACT

PAGES IPO: pages@pages.unibe.ch

REFERENCE

PAGES IPO (2014) PAGES Mag 22: 3 